\title{
Minimization of Ohmic Losses for Domain Wall Motion in a Ferromagnetic Nanowire
}

\author{
O. A. Tretiakov, Y. Liu, and Ar. Abanov \\ Department of Physics, MS 4242, Texas A\&M University, College Station, Texas 77843-4242, USA
}

(Received 31 May 2010; published 15 November 2010)

\begin{abstract}
We study current-induced domain-wall motion in a narrow ferromagnetic wire. We propose a way to move domain walls with a resonant time-dependent current which dramatically decreases the Ohmic losses in the wire and allows driving of the domain wall with higher speed without burning the wire. For any domain-wall velocity we find the time dependence of the current needed to minimize the Ohmic losses. Below a critical domain-wall velocity specified by the parameters of the wire the minimal Ohmic losses are achieved by dc current. Furthermore, we identify the wire parameters for which the losses reduction from its dc value is the most dramatic.
\end{abstract}

DOI: $10.1103 /$ PhysRevLett.105.217203

PACS numbers: 75.78.Fg, 75.60.Ch, 85.75.- d

Introduction.-In recent years there has been intense interest in applications of domain-wall (DW) motion in ferromagnetic nanowires [1,2]. This interest is mostly based on the possibility to store and exchange information by means of moving domain walls which separate the regions of magnetization parallel and antiparallel to the wire. These regions with parallel and antiparallel magnetization can be thought of as two bits, zero and one, of binary information storage.

DWs can be moved by a magnetic field $[1,3]$ or electric current $[2,4]$. For technological applications the current driving is preferred as a magnetic field is difficult to apply locally to small wires. Thus, in this Letter we consider the current-driven DW devices. To achieve their highest performance it is important to minimize the losses on Joule heating in the wire, which are due to the resistance of the wire itself and the entire circuit. They are proportional to the time-averaged current square, $\left\langle J^{2}\right\rangle$. Their minimization has a twofold advantage. First, one can increase the maximum current which still does not destroy the wire by excessive heating and therefore move the DWs with a higher velocity, since the DW velocity increases with the applied current. Second, it creates the most energy efficient memory devices and also increases their reliability.

To achieve these goals we propose to utilize a "resonant" time-dependent current, which allows us to gain a significant reduction of Ohmic losses. We show that all thin wires can be characterized by three parameters obtained from dc-driven DW motion experiments: critical current $j_{c}$, drift velocity at the critical current $V_{c}$, and the material dependent parameter $a>0$, which, in particular, depends on Gilbert damping $\alpha$ and nonadiabatic spin torque constant $\beta$. The parameter $a$ is just a ratio of the slopes of the drift velocity $V_{d}(J)$ at large and small dc currents; see the upper inset of Fig. 1. Our main results are summarized in Fig. 2. We find the minimal power $\left\langle J^{2}\right\rangle$ needed to drive a DW with drift velocity $V_{d}$. Figures 2(a) and 2(b) show the dependence of power $\left\langle J^{2}\right\rangle$ on $V_{d}$ for the optimal time-dependent current-red solid curves, and for dc current-black dashed curves, for two cases: (a) $a<1$ and (b) $a>1$. In Fig. 2(a) the minimal power is given by dc current for $V_{d}<V_{c}$, but above $V_{c}$ there is a significant reduction in the heating power compared to dc current. Figure 2(b) shows that the power $\left\langle J^{2}\right\rangle$ is reduced in comparison with the dc case for $V_{d}>V_{c} v_{\text {rc }}$. The (dimensionless) resonant critical velocity $v_{\text {rc }} \leq 1$ and can be extracted from the dc-current measurements. For Permalloy using [5] we estimated $a \approx 0.5$, see Fig. 2(a), where for $V_{d} \gtrsim V_{c}$ the power is less than $50 \%$ of that for the dc current.

Figures 2(c) and 2(d) show the limiting cases of $a \ll 1$ (c) and $a \gg 1$ (d). We note that for small $\alpha$ and $\beta$,

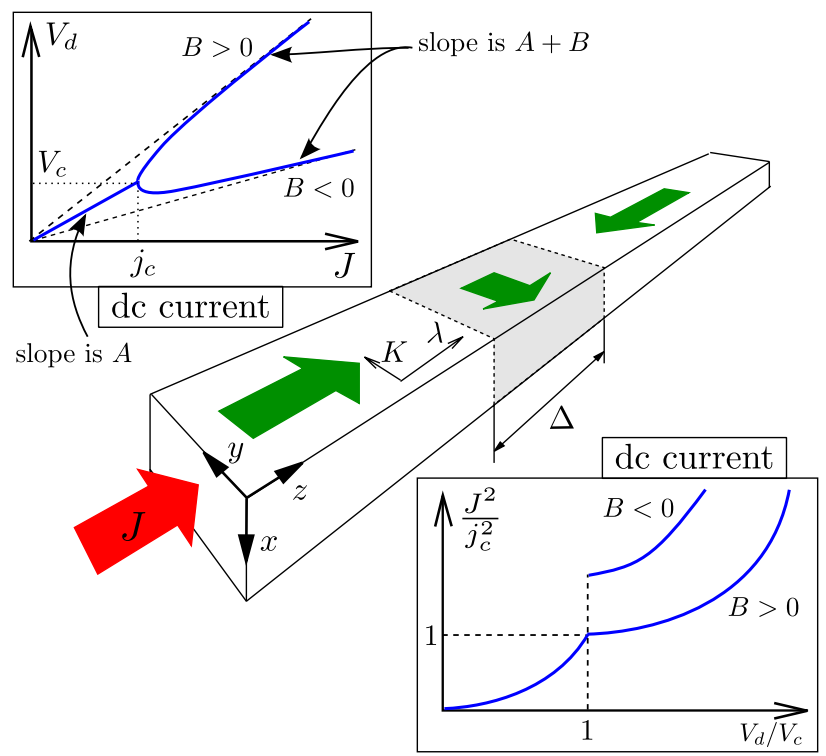

FIG. 1 (color online). A sketch of a current-driven domain wall in the ferromagnetic wire. The upper inset shows the dependence of drift velocity $V_{d}$ of DW on dc current $J$ for $B>0$ and $B<0$, see Eq. (2b). The slope at $J<j_{c}$ is given by $A$, while at $J \gg j_{c}$ it is $A+B$. The lower inset shows the power of Ohmic losses $p_{\mathrm{dc}}\left(V_{d} / V_{c}\right)=J^{2} / j_{c}^{2}$ for dc current. For $B<0$ the power has a discontinuity at $V_{d} / V_{c}=1$. 

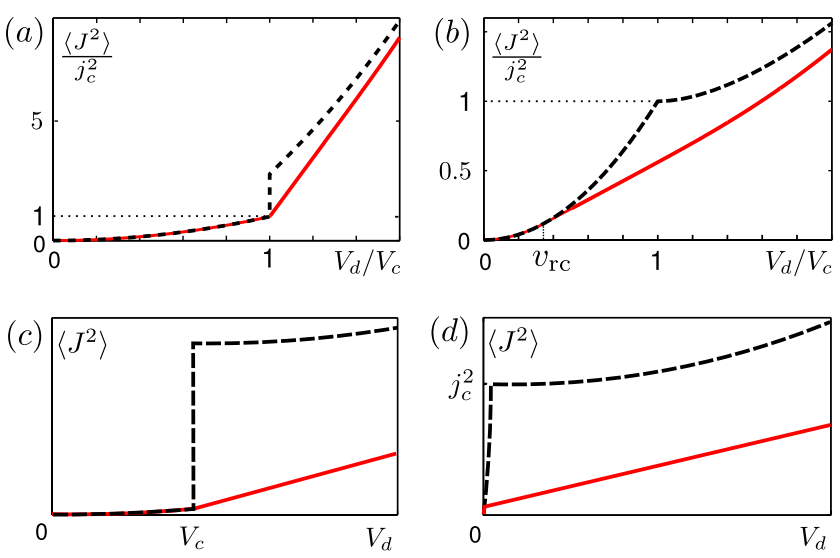

FIG. 2 (color online). Minimal power of Ohmic losses $\bar{p}=$ $\left\langle J^{2}\right\rangle / j_{c}^{2}$ as a function of drift velocity $V_{d}$ shown by solid line for (a) $a=0.5$ (b) $a=2$. The dashed line depicts $\bar{p}$ for dc current. A sketch of $\left\langle J^{2}\right\rangle\left(V_{d}\right)$ shown by solid line in (c) for $\beta \gg \alpha(a \ll 1)$ and (d) for $\beta \ll \alpha(a \gg 1)$.

$a \approx \alpha / \beta$. If $a \ll 1(\beta \gg \alpha)$, Fig. 2(c), for dc current the excessive heating power $\sim 1 / a^{2}$ essentially limits the highest achievable drift velocity $V_{d}$ by $V_{c}$, whereas the resonant ac current can move DWs with much higher $V_{d}$ (and still rather low power). In the opposite case $a \gg 1,(\beta \ll \alpha)$, Fig. 2(d), the power saving starts to be considerable at very small velocity $V_{d}$. If $\beta=0$ the dc-current power is finite even at $V_{d} \rightarrow 0$, while for the resonant ac current the power linearly approaches zero at small $V_{d}$. Therefore, our approach gives a dramatic power reduction even in the least favorable cases $\beta \ll \alpha$ and $\beta \gg \alpha$, thus opening new doors for using materials with much wider range of $\beta$ for fast DW motion.

Model.-DW in a ferromagnetic wire can be modeled by a Hamiltonian which contains exchange and dipolar interactions. In a thin wire, the latter can be approximated by two anisotropies: along the wire $(\lambda)$ and transverse to it $(K)$. A sketch of a wire with a DW of width $\Delta$ is shown in Fig. 1. The dynamics of magnetization $\mathbf{S}$ in a wire is described by Landau-Lifshitz-Gilbert (LLG) equation with the current $J[6,7]$,

$$
\dot{\mathbf{S}}=\mathbf{S} \times \mathbf{H}_{e}-J \partial \mathbf{S}+\beta J \mathbf{S} \times \partial \mathbf{S}+\alpha \mathbf{S} \times \dot{\mathbf{S}},
$$

where $\mathbf{H}_{e}=\delta \mathcal{H} / \delta \mathbf{S}$ is the effective magnetic field given by the Hamiltonian $\mathcal{H}$ of the system, $\alpha$ is Gilbert damping constant, $\beta$ is nonadiabatic spin torque constant, and $\partial=$ $\partial / \partial z$. Furthermore, it can be shown [8] that in a thin wire the DW is a rigid spin texture for not too strong applied currents and its dynamics can be described in terms of only two collective coordinates (corresponding to the two softest modes of the DW motion): namely, the position of the DW along the wire $z_{0}$ and the rotation angle $\phi$ of the magnetization in the DW around the wire axis.

To describe the DW dynamics we need to find the equations of motion. For the two softest modes of the $\mathrm{DW}, z_{0}(t)$ and $\phi(t)$, they can be found as an expansion in small current $J$ up to a linear in $J$ order. Because of the translational invariance $\dot{z}_{0}$ and $\dot{\phi}$ cannot depend on $z_{0}$. In addition, to the first order in small transverse anisotropy $K$, $\dot{\phi}$ and $\dot{z}_{0}$ are proportional to the first harmonic $\sin (2 \phi)$. Then the most general equations of DW motion are

$$
\begin{aligned}
\dot{\phi} & =C\left[J-j_{c} \sin (2 \phi)\right], \\
\dot{z}_{0} & =A J+B\left[J-j_{c} \sin (2 \phi)\right],
\end{aligned}
$$

where $J(t)$ is, in general, a time-dependent current whose frequency is not too high to create spin waves and other excitations in the wire. Coefficients $A, B, C$, and critical current $j_{c}$ can be calculated for a particular model [9] in terms of $\alpha, \beta$ and other microscopic parameters by means of deriving Eqs. (2) from the LLG Eq. (1). However, we emphasize that Eqs. (2), with coefficients $A, B, C$, and $j_{c}$ determined directly from dc-current experiment for each particular wire, have more general validity than just being derived from LLG, e.g., due to the complicated influence of disorder and internal DW dynamics [10]. Namely, the value of $j_{c}$ is defined as the endpoint of the linear regime of the time-averaged (drift) velocity $V_{d}=\left\langle\dot{z}_{0}(J)\right\rangle$; see the upper inset of Fig. 1. The linear slope of $V_{d}(J)$ below $j_{c}$ determines constant $A$. The slope of $V_{d}(J)$ at large $J$ gives $A+B$. Constant $C$ one can obtain, e.g., from the measurements of the DW electromotive force [11,12] for dc current.

$d c$ current.-For the dc current applied to the wire the DW dynamics governed by Eqs. (2) can be obtained explicitly [8]. For $J<j_{c}$ and $A \neq 0$ the DW moves along the wire but does not rotate around its axis. It only tilts on angle $\phi_{0}$ from the transverse-anisotropy easy axis ( $y$ axis) given by condition $\sin \left(2 \phi_{0}\right)=J / j_{c}$. The drift velocity is given by $V_{d}=A J$; see Eq. (2b). At $J=j_{c}$ the magnetization angle becomes perpendicular to the easy axis, $\phi_{0}=$ $\pi / 2$. For $J>j_{c}$ the DW both moves and rotates, and Eqs. (2) give $V_{d}=A J+B \sqrt{J^{2}-j_{c}^{2}}$ [8].

The influence of the spin structure on the current is negligible. The largest losses in the system are the Ohmic losses of the current. The power of Ohmic losses is proportional to $J^{2}$. Therefore, at $J<j_{c}$ the current is $J=V_{d} / A$ and the power of Ohmic losses is $\mathcal{P}_{\mathrm{dc}}=J^{2}=V_{d}^{2} / A^{2}$. It is instructive to introduce the dimensionless variables for time, drift velocity, current, and power. Using $V_{c}=A j_{c} \simeq$ $K \Delta$ we find [13]

$$
\tau=C j_{c} t, \quad v_{d}=V_{d} / V_{c}, \quad j=J / j_{c}, \quad p=\mathcal{P} / j_{c}^{2} .
$$

Using Eq. (3) we find $p_{\mathrm{dc}}=v_{d}^{2}$ for $v_{d}<1$.

For currents above $j_{c}$ the dimensionless power $p_{\mathrm{dc}}$ is given in terms of dimensionless drift velocity $v_{d}=j+$ $(B / A) \sqrt{j^{2}-1}$ as $p_{\mathrm{dc}}\left(v_{d}\right)=j^{2}$, see the lower inset of Fig. 1. Thus, it is quadratic in $v_{d}$, and at $v_{d} \gg 1$ it approaches $p_{\mathrm{dc}}=A^{2} v_{d}^{2} /(B+A)^{2}+B /(B+A)$. For $B>$ 0 right above $v_{d}=1$, it is approximated by $p_{\mathrm{dc}}=1+$ $(A / B)^{2}\left(v_{d}-1\right)^{2}$. For $B<0$ the power has a discontinuity at $v_{d}=1$. 
Minimization of Ohmic losses by time-dependent current.-In this part we minimize the Ohmic losses while keeping the DW moving with a given drift (average) velocity. Equations of motion (2) are correct even when the current depends on time. In general, the DW motion has some period $T$ and current $j(\tau)$ must be a periodic function with the same $T$ to minimize the Ohmic losses.

In the following it is more convenient to measure the angle from the hard axis instead of easy axis and to scale it by factor of 2 , so that $2 \phi=\theta-\pi / 2$. Also, we introduce the ratio of slopes of $V_{d}(J)$ at large and small currents $a=(A+B) / A$. Then using Eq. (2a) the dimensionless current becomes

$$
j(\tau)=\dot{\theta} / 2-\cos \theta,
$$

where $\dot{\theta}=\partial \theta / \partial \tau$. Averaging Eq. (2b) over dimensionless period $T$ we find

$$
v_{d}=\frac{a}{2}\langle\dot{\theta}\rangle-\langle\cos \theta\rangle,
$$

where $\langle\ldots\rangle=\frac{1}{T} \int_{0}^{T} \ldots d \tau$ is the time averaging.

To minimize the power of Ohmic losses $\bar{p}$ averaged over time we need to find the minimum of $\left\langle j^{2}(\tau)\right\rangle$ at fixed $v_{d}$ given by Eq. (5),

$$
\bar{p}=\left\langle\left(\frac{\dot{\theta}}{2}-\cos \theta\right)^{2}-2 \rho\left(\frac{a}{2} \dot{\theta}-\cos \theta-v_{d}\right)\right\rangle .
$$

Here, to account for the constraint given by Eq. (5), we used a Lagrange multiplier $2 \rho$, with $\rho$ being an arbitrary dimensionless constant. Note that the cross term $\sim \int \dot{\theta} \cos \theta d \tau^{\prime}$ and the term $\sim \int \dot{\theta} d \tau^{\prime}$ can be dropped for the minimization procedure as they are full derivatives.

Power (6) can be considered as an effective action for a hypothetical particle of mass $1 / 2$ in a periodic potential field, and its minimization leads to the equation of motion

$$
\frac{\ddot{\theta}}{2}=-\frac{\partial U}{\partial \theta}, \quad U(\theta, \rho)=-\cos ^{2} \theta-2 \rho \cos \theta .
$$

It can be reduced to the first order differential equation

$$
\dot{\theta}= \pm 2 \sqrt{d-U(\theta, \rho)},
$$

where $d$ is an arbitrary integration constant. Note that changing $\rho \rightarrow-\rho$ in $U$ of Eq. (7) is equivalent to changing $\theta \rightarrow \pi+\theta$, so below we consider only positive $\rho$. The potential has a minimum at $\theta=0$ with $U_{\min }=2 \rho-1$ for any $\rho \geq 0$. For $\rho<1$ it has also minimum at $\theta= \pm \pi$ with $U( \pm \pi)=-2 \rho-1$ and the maximum at $\cos \theta_{\rho}=-\rho$ with $U\left( \pm \theta_{\rho}\right)=\rho^{2}$. For $\rho>1$ it has maximum at $\theta= \pm \pi$ with $U( \pm \pi)=2 \rho-1$.

According to Eq. (8) there are two different regimes: (i) the rocking regime where $d<\max [U(\theta, \rho)]$ in which case $\theta$ is bounded, and the particle oscillates in potential well $U(\theta)$, see Fig. 3; and (ii) the rotational regime where $d>\max [U(\theta, \rho)]$ in which case the magnetization in the DW rotates. Below we consider these regimes separately.
Rocking regime.-In this regime the motion of $\theta$ mimics pendulum motion. The particle rocks between the two turning points $-\theta_{0}$ and $\theta_{0}$ given by the condition $d=$ $U\left( \pm \theta_{0}, \rho\right)$. At these points $\dot{\theta}=0$. Since $\theta$ is a bounded function $\langle\dot{\theta}\rangle=0$ and the averaged velocity becomes $v_{d}=-\langle\cos \theta\rangle$. The averaging is done over a period of one complete oscillation,

$$
T=\int_{-\theta_{0}}^{\theta_{0}} \frac{d \theta}{\sqrt{d-U(\theta, \rho)}}
$$

and according to Eq. (6) the power is given by

$$
\bar{p}=\left\langle\dot{\theta}^{2}\right\rangle / 4+\left\langle\cos ^{2} \theta\right\rangle .
$$

Most generally $\theta$ depends on time. For any $\theta(\tau)$, however, $\left\langle\cos ^{2} \theta\right\rangle \geq\langle\cos \theta\rangle^{2}$. Then from Eq. (10) follows $\bar{p} \geq$ $\left\langle\dot{\theta}^{2}\right\rangle / 4+\langle\cos \theta\rangle^{2}=\left\langle\dot{\theta}^{2}\right\rangle / 4+v_{d}^{2} \geq v_{d}^{2}=p_{\mathrm{dc}}$. Thus, in the bounded regime the power of Ohmic losses is minimal for dc current and is given by $\bar{p}=v_{d}^{2}$.

Rotational regime.-Next we study the case when $d>$ $\max [U(\theta, \rho)]$, so that angle $\theta$ is unbounded. It corresponds to the rotational motion of the transverse to the wire component of the DW magnetization. Note that in the rotational regime the term in Eq. (5) with $\langle\dot{\theta}\rangle$ should be kept because $\theta$ is not bounded. The time it takes for $\theta$ to make a full rotation from $-\pi$ to $\pi$ defines the period $T$. Then the period, drift velocity, and power, according to Eq. (10), are given by

$$
\begin{aligned}
T & =\frac{1}{2} \int_{-\pi}^{\pi} \frac{d \theta}{\sqrt{d-U(\theta, \rho)}}, \\
v_{d} & =\frac{\pi a}{T}-\frac{1}{2 T} \int_{-\pi}^{\pi} \frac{\cos \theta d \theta}{\sqrt{d-U(\theta, \rho)}}, \\
\bar{p} & =\frac{1}{2 T} \int_{-\pi}^{\pi} \frac{d-U(\theta, \rho)+\cos ^{2} \theta}{\sqrt{d-U(\theta, \rho)}} d \theta .
\end{aligned}
$$

This system of equations, after minimizing the power $\bar{p}$ with respect to both $d$ and $\rho$ at fixed $v_{d}$, gives $\bar{p}\left(v_{d}\right)$. One can either directly perform a numerical minimization of Eq. (11) or alternatively try to find the minimization condition for $\bar{p}$ analytically. We have followed both routes.

The minimization of Eqs. (11) infers that $\partial \bar{p} /\left.\partial \rho\right|_{v_{d}}=0$ from which one can find [15]

$$
\int_{-\pi}^{\pi} \sqrt{d-U(\theta, \rho)} d \theta=2 \pi a \rho .
$$

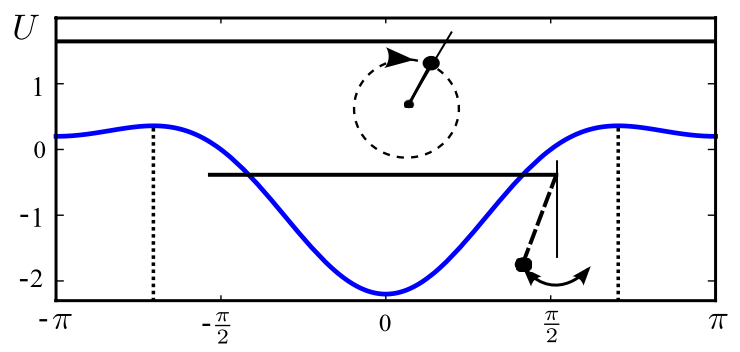

FIG. 3 (color online). Potential $U(\theta)$ in which a "particle" is moving in the rocking (pendulumlike) and rotational regimes. 
This equation gives the relationship between $d$ and $\rho$. Solving it together with Eq. (11b) one finds $d$ and $\rho$ in terms of $v_{d}$. They are then substituted into $\bar{p}=2 \rho v_{d}-d$ which follows from Eqs. (11c) and (12). The motion is unbounded when $d>\max [U(\theta, \rho)]$, see Eq. (8), which leads to $d>\rho^{2}$ for $\rho<1$ and $d>2 \rho-1$ for $\rho>1$.

The results for the minimal power of Ohmic losses $\bar{p}\left(v_{d}\right)$ are presented in Fig. 2. For $a<1$, see, e.g., Fig. 2(a), at $v_{d}<v_{\mathrm{rc}}=1$ the minimal power $\bar{p}$ coincides with the one given by dc current, whereas at $v_{d}>1$ it is significantly lower than $p_{\mathrm{dc}}$. Immediately above $v_{d}=1$ we find that there is a range of $v_{d}$ where $\bar{p}=1+2 \rho_{0}\left(v_{d}-1\right)$ with $\rho_{0}(a)>1$ given by Eq. (12) with $d=2 \rho-1$. Therefore, $\bar{p}$ is linear in $v_{d}$ right above $v_{\mathrm{rc}}=1$.

For $a>1$, see, e.g., Fig. 2(b), we show [15] that there is a critical velocity $v_{\text {rc }}<1$, such that at $v_{d}<v_{\text {rc }}$ the power of Ohmic losses is $\bar{p}=v_{d}^{2}=p_{\mathrm{dc}}$. Above $v_{\text {rc }}$ one can minimize the Ohmic losses by moving DW with resonant current pulses. Right above $v_{\text {rc }}$ there is a certain range of $v_{d}$ where $d \simeq \rho^{2}$, and therefore we find $\bar{p}=2 \rho_{0} v_{d}-\rho_{0}^{2}$ with $\rho_{0}(a)<1$ given by Eq. (12) with $d=\rho^{2}$. The critical velocity is found as $v_{\mathrm{rc}}=\rho_{0}(a)$. For $a \gg 1$ [corresponding to nonadiabatic spin transfer torque coefficient $\beta \ll \alpha$, cf. Eq. (1)] we find $v_{\text {rc }} \simeq 2 /(\pi a)$ and therefore for $v_{d} \gg v_{\text {rc }}$ we obtain $\bar{p}=4 v_{d} /(\pi a)$.

We show that at large $v_{d}$ the minimal power is always smaller than $p_{\mathrm{dc}}$. Note that for $d \gg 1$ Eq. (12) gives $d=a^{2} \rho^{2}$. Using it we find that the difference between them approaches $p_{\mathrm{dc}}-\bar{p}=(1-1 / a)^{2} / 2$ at $v_{d} \gg 1$.

Optimal current.-For $v_{d}<v_{\text {rc }}$ the optimal current coincides with the dc current. Above $v_{\text {rc }}$ the resonant current $j(t)$ is plotted in Fig. 4 for different velocities $v_{d}$ in the case $a=2$. At small $v_{d}$ the current is given by $j(\tau)=-2 \cos (\theta(\tau))-v_{\mathrm{rc}}$ for $\cos (\theta(\tau))<-v_{\mathrm{rc}}$ and by $j(\tau)=v_{\text {rc }}$ for $\cos (\theta(\tau))>-v_{\text {rc }}$. At $v_{d} \gg 1$ the current is approximated by $j \approx v_{d} / a+[(1-a) / a] \cos \theta$.

In general, at $v_{d}>v_{\text {rc }}$ the current's maximum $j_{\max }$ increases from $2-v_{\text {rc }}$ at small enough $v_{d} \lesssim 1$ up to $j_{\max } \approx v_{d} / a$ at $v_{d} \gg 1$. The current's minimum increases monotonically from small positive values $j_{\min }=v_{\text {rc }}$ at $v_{d} \sim 1$ up to $j_{\min }=j_{\max }-2|1-a| / a$ at $v_{d} \gg 1$. At $v_{d} \lesssim 1$ (for $a>1$ ) the time between the current picks decreases with increasing velocity as $T \simeq(\pi a-$ $\left.2 \arcsin v_{\text {rc }}\right) /\left(v_{d}-v_{\text {rc }}\right)$, whereas the pick's width is given by $\approx 1.3 / \sqrt{\left(1-v_{\mathrm{rc}}\right)}$, which is independent of $v_{d}$. Therefore, at small $v_{d}-v_{\text {rc }}$ the picks are widely separated, then as $v_{d}$ increases the time between the picks decreases. At $v_{d} \gg 1$ the optimal current has a large constant component, which is close to but smaller than the dc current for the same $v_{d}$, and has small-amplitude ac modulations with a period $T \approx \pi a / v_{d}$ on top of it.

Summary.-We have studied the current-driven DW dynamics in thin ferromagnetic wires. We have found the ultimate lower bound for the Ohmic losses in the wire for any DW drift velocity $V_{d}$. The explicit time dependence of current, see Fig. 4, has been found which minimizes the

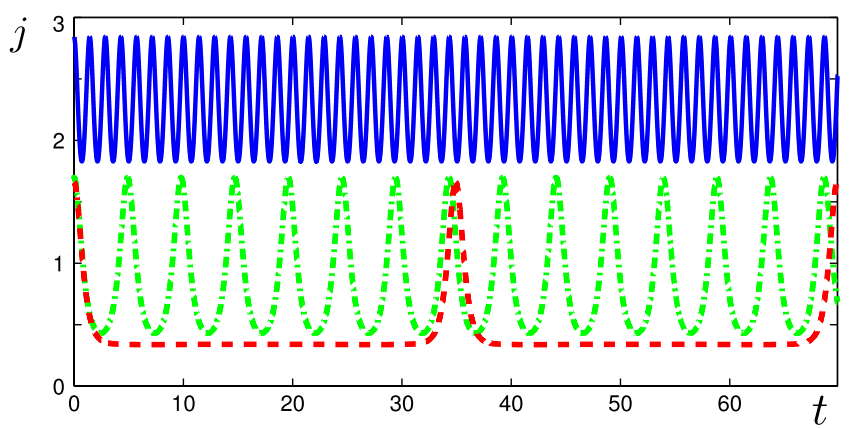

FIG. 4 (color online). Current $j$ as a function of time $\tau$ at velocities $v_{d}=0.5$ (dashed line), $v_{d}=1.5$ (dot-dashed line), and $v_{d}=4.5$ (solid line) in the rotational regime for $a=2$.

Ohmic losses. We have shown that the use of these specific current pulses instead of applying dc current can help to significantly reduce heating of the wire for any $V_{d}$. Even in the limiting cases of the systems with weak $(\beta \ll \alpha)$ or strong $(\beta \gg \alpha)$ nonadiabatic spin transfer torque, where the power of Ohmic losses is high for dc currents, the optimized ac current gives significant reduction in heating power thus greatly expanding the range of materials which can be used for spintronic devices $[1,2]$.

We are grateful to J. Sinova for valuable discussions. This work was supported by the NSF Grant No. 0757992 and the Welch Foundation (A-1678).

[1] D. A. Allwood et al., Science 309, 1688 (2005).

[2] S. S. P. Parkin, M. Hayashi, and L. Thomas, Science 320, 190 (2008); M. Hayashi et al., Science 320, 209 (2008).

[3] T. Ono et al., Science 284, 468 (1999).

[4] A. Yamaguchi et al., Phys. Rev. Lett. 92, 077205 (2004).

[5] R. Moriya et al., Nature Phys. 4, 368 (2008).

[6] Z. Li and S. Zhang, Phys. Rev. Lett. 92, 207203 (2004).

[7] A. Thiaville et al., Europhys. Lett. 69, 990 (2005).

[8] O.A. Tretiakov and Ar. Abanov, Phys. Rev. Lett. 105, 157201 (2010).

[9] As it was shown in Ref. [8], $A=\beta / \alpha, B=(\alpha-\beta) \times$ $(1+\alpha \Gamma \Delta) /\left[\alpha\left(1+\alpha^{2}\right)\right], \quad C=(\alpha-\beta) \Delta /\left[\left(1+\alpha^{2}\right) \Delta_{0}^{2}\right]$, and $j_{c}=(\alpha K \Delta /|\alpha-\beta|)[\pi \Gamma \Delta / \sinh (\pi \Gamma \Delta)]$. Here $\Gamma=$ $D / J_{\text {ex }}, \quad J_{\text {ex }}$ is the exchange constant, and $D$ is the Dzyaloshinskii-Moriya interaction (DMI) constant. Also, $\Delta=\Delta_{0} / \sqrt{1-\Gamma^{2} \Delta_{0}^{2}}$, where $\Delta_{0}$ is the DW width in the absence of DMI.

[10] H. Min et al., Phys. Rev. Lett. 104, 217201 (2010).

[11] S. A. Yang et al., Phys. Rev. Lett. 102, 067201 (2009).

[12] Y. Liu, O. Tretiakov, and Ar. Abanov (unpublished).

[13] It can be shown that $C \sim B \sim \alpha-\beta$ [8]. In the special case of $\alpha=\beta$, we find $C=B=0$ and one cannot use dimensionless variables (3). The DW dynamics in this case is trivial [14]. The DW does not rotate $\dot{\phi}=0$ and moves with the velocity given by current $\dot{z}_{0}=J$.

[14] S. E. Barnes and S. Maekawa, Phys. Rev. Lett. 95, 107204 (2005).

[15] See supplementary material at http://link.aps.org/ supplemental/10.1103/PhysRevLett.105.217203 for the method description. 